\title{
Ontogenetic functional diversity: Size structure of a keystone predator drives functioning of a complex ecosystem
}

\author{
Volker H. W. Rudolf ${ }^{1}$ AND Nick L. Rasmussen \\ Department of Ecology and Evolutionary Biology, Rice University, 6100 Main Street, Houston, Texas 77005 USA
}

\begin{abstract}
A central challenge in community ecology is to understand the connection between biodiversity and the functioning of ecosystems. While traditional approaches have largely focused on species-level diversity, increasing evidence indicates that there exists substantial ecological diversity among individuals within species. By far, the largest source of this intraspecific diversity stems from variation among individuals in ontogenetic stage and size. Although such ontogenetic shifts are ubiquitous in natural communities, whether and how they scale up to influence the structure and functioning of complex ecosystems is largely unknown. Here we take an experimental approach to examine the consequences of ontogenetic niche shifts for the structure of communities and ecosystem processes. In particular we experimentally manipulated the stage structure in a keystone predator, larvae of the dragonfly Anax junius, in complex experimental pond communities to test whether changes in the population stage or size structure of a keystone species scale up to alter community structure and ecosystem processes, and how functional differences scale with relative differences in size among stages.

We found that the functional role of $A$. junius was stage-specific. Altering what stages were present in a pond led to concurrent changes in community structure, primary producer biomass (periphyton and phytoplankton), and ultimately altered ecosystem processes (respiration and net primary productivity), indicating a strong, but stage-specific, trophic cascade. Interestingly, the stage-specific effects did not simply scale with size or biomass of the predator, but instead indicated clear ontogenetic niche shifts in ecological interactions. Thus, functional differences among stages within a keystone species scaled up to alter the functioning of entire ecosystems. Therefore, our results indicate that the classical approach of assuming an average functional role of a species can be misleading because functional roles are dynamic and will change with shifts in the stage structure of the species. In general this emphasizes the importance of accounting for functional diversity below the species level to predict how natural and anthropogenic changes alter the functioning of natural ecosystems.
\end{abstract}

Key words: Anax junius; dragonfly larvae; ecosystem functioning; food web; functional diversity; ontogenetic niche shift; size structure.

\section{INTRODUCTION}

Linking biodiversity to the structure and functioning of natural ecosystems has been a longstanding challenge in ecology. While much research has examined the impacts of species diversity on the functioning of ecosystems (McCann 2000, Loreau et al. 2001, Hooper et al. 2005, Duffy et al. 2007), variation below the species level is typically ignored in these studies (Reiss et al. 2009, Rudolf and Lafferty 2011). This reflects the classical approach to community ecology, which is based on the premise that we can predict the dynamics of communities by assuming that all individuals within a species are functionally identical. However, substantial evidence indicates that ecological variation among individuals within a species often exceeds variation

Manuscript received 7 March 2012; revised 26 November 2012; accepted 26 November 2012. Corresponding Editor: H. Hillebrand.

${ }^{1}$ E-mail: volker.rudolf@rice.edu among species (Polis 1984, Munoz and Ojeda 1998, Woodward and Hildrew 2002, Bolnick et al. 2003, Harmon et al. 2009). The unresolved question is whether this variation matters at the community and ecosystem scale in complex natural ecosystems.

By far the largest source of this intraspecific variation between individuals stems from differences in ontogenetic stage and size (Polis 1984, Werner and Gilliam 1984, Lomnicki 1988, Persson 1999, Benton et al. 2006). Individuals often experience substantial changes in their ecological role during their ontogeny. These changes are also called ontogenetic niche shifts and include shifts in habitat use, diet and resource use, predation risk, foraging rate, reproductive status, or other key life history traits (reviewed in Wilbur 1980, Werner and Gilliam 1984, Yang and Rudolf 2010). For instance, many predatory fish such as Eurasian perch forage in the littoral zone of lakes on zooplankton and invertebrates during the juvenile stage, but move to the pelagic zone to prey on other fish once they become mature (Mittelbach 
and Persson 1998). Ontogenetic niche shifts occur in $>80 \%$ of animal taxa (Werner 1988) and a recent analysis indicates that the average resource overlap among stages of a species can range from $0 \%$ to $100 \%$ and be as low as $37 \%$ for entire ecological networks (Rudolf and Lafferty 2011). Plants experience similar changes in species interactions (e.g., due to changes in resistance to herbivory) during ontogeny (Boege and Marquis 2005, Yang and Rudolf 2010). Consequently, ontogenetic niche shifts are ubiquitous in natural communities (Rudolf and Lafferty 2011). While increasing evidence indicates the importance of ontogenetic niche shifts for the dynamics of species interactions (reviewed in Miller and Rudolf 2011), the consequences for the structure and functioning of complex ecosystems are largely unknown.

With ontogenetic niche shifts, stages within species often differ substantially in their ecological interactions and thus may also differ in their functional role. Given such functional differences across ontogenetic stages, any changes in the population size structure could strongly alter the functional role of a species and therefore its impact on the ecosystem. The size structure of populations often varies considerably within a season (Wissinger 1988, 1992, Urban 2007, Rudolf 2012), or over years and decades (Osenberg et al. 1992, Persson et al. 2003), or differs between populations in different habitats (Osenberg et al. 1992) due to changes (natural or anthropogenic) in environmental conditions. Thus, understanding the consequences of this ontogenetic diversity for the dynamics of communities and ecosystem processes is critical to understand the functional role of species in natural communities.

Predicting whether and how changes in the size structure of a species affect the structure of communities and ecosystem processes is not straightforward. For instance, per capita consumption rates (and thus interaction strength) are often expected to increase with size (Yodzis and Innes 1992, Emmerson and Raffaelli 2004). Large stages often have broader diets than small stages (with diets of small stages frequently nested within diets of large stages) and face lower predation risks (reviewed in Woodward et al. 2005). In addition, in predatory species, larger stages also typically have a higher trophic position, and top predators often have disproportionally strong effects on the community (Power et al. 1996). All of these relationships would suggest that the largest stage has the strongest and broadest impact on the ecosystem (thus largely determining the functional role of a species in the ecosystem). On the other hand, per-unit-biomass consumption rates and population density are often expected to decrease with size (Reiss et al. 2011). If strong enough, this negative relationship between body size and per-biomass consumption rate could weaken or even reverse the relative impact of stages on the ecosystem, with smaller stages having the strongest impact on the system. In both scenarios, the functional differences among the stages scale positively with differences in size. However, if ontogenetic niche shifts in species interactions occur (e.g., small stages may interact with a completely different part of the community than large stages), we may not see a clear relationship (positive or negative) between size and relative impact on the ecosystem. In the latter scenario, the functional role of a species would show clear shifts with changes in the size structure.

Here we take an experimental approach to examine whether and how stages differ in their functional role by manipulating the size structure of a keystone predator, the larval stage of the dragonfly Anax junius, in experimental pond communities. In particular we test (1) whether changes in the population size structure of a keystone species alter its functional role in the ecosystem, (2) whether these changes scale up to alter community structure and ecosystem processes, and (3) how functional differences scale with relative differences in size among stages.

\section{Methods \\ Focal organisms}

Several aspects of larvae of the dragonfly Anax junius (see Plate 1) create an excellent system for studying the ecological consequences of ontogenetic functional diversity. First, this generalist predator is one of the most abundant and widespread dragonflies in North America and is known to strongly impact the structure of fishless pond communities (Van Buskirk 1988, Wilbur and Fauth 1990, McPeek 1998). Secondly, individuals increase more than 17 -fold in length during development and the stage structure of the population changes during the course of a season (Wissinger 1992; V. H. W. Rudolf, personal observation). Third, our preliminary stable isotope analysis of natural populations indicates that its trophic position strongly increases with size (V. H. W. Rudolf, unpublished data).

\section{Experimental design}

Detecting functional differences among very differentsized predators is challenging because individuals differ substantially in their biomass. In traditional designs, predator densities or biomass are often standardized. However, when predators differ substantially in size, such substitutive designs lead to dramatic differences in either biomass or density and conclusions about identity effects are likely to be confounded by these differences (Chalcraft and Resetarits 2004, O'Connor et al. 2008). Such confounding effects can be even more amplified when individual growth rates (and mortality rates) are higher in smaller individuals (as in our study species), because treatments would rapidly diverge through time. Furthermore, neither density nor total biomass is ever constant across stage classes within in our study species (Wissinger 1992; V. H. W. Rudolf, unpublished data), and keeping total biomass constant would lead to unrealistically high densities of small stages and experimental artifacts. Consequently, in systems where 
the effects of individuals on the ecosystem are largely driven by body size, there is a high risk of misinterpreting results when using traditional substitutive designs (Chalcraft and Resetarits 2004, Schneider et al. 2012). Therefore, we refrained from keeping initial biomass or density constant and followed previous suggestions that recommend using natural size-abundance relationships (Chalcraft and Resetarits 2004, O'Connor et al. 2008). This approach allowed us to estimate the actual impact of each size class in natural populations and the relative impact of each size class by separating quantitative differences from qualitative differences among size classes.

The experiment consisted of four treatments, each replicated six times $(n=24)$ : ponds received 18 small $A$. junius (head width (HW) 2.85-3.3 mm, body length (BL) 8.9-11.7 mm, dry mass (DM) $0.026-0.04 \mathrm{mg}$ ), or 6 medium A. junius $(\mathrm{HW} \sim 4.5 \mathrm{~mm}, \mathrm{BL} \sim 16 \mathrm{~mm}, \mathrm{DM}$ $\sim 0.24 \mathrm{mg}$ ), or 3 large $A$. junius ( $\mathrm{HW} \sim 6.9 \mathrm{~mm}, \mathrm{BL} \sim 32$ $\mathrm{mm}, \mathrm{DM} \sim 0.61 \mathrm{mg}$ ), or none of these stages (control). We collected all $A$. junius larvae from one local pond. Size classes were chosen based on the size structure of the natural population and to maximize differences among size classes while reducing the risk of early metamorphosis of the largest size class. Density and relative abundance of each size class were a direct reflection of the relative size-abundance relationship of the natural A. junius population (V. H. W. Rudolf, unpublished data). Importantly, differences in initial total biomass across treatments strongly declined during the course of the experiment (due to natural differences in growth rates among stages), and there were no significant differences among average biomass in small and large predator treatments (see Appendix B for details). The experiment was terminated after three weeks when the first $A$. junius larvae started to emerge as adults.

\section{Experimental pond communities}

The experiment was carried out in experimental outdoor ponds that closely mimicked the structure and complexity of local fishless ponds. Full details of the experimental procedures are given in Appendix A. In brief, experimental ponds were established in 1200-L plastic stock tanks set up on an open field in a randomized complete-block design three months before the start of the experiment. Each tank was fitted with a $50 \%$ shade cloth lid and received $220 \mathrm{~g}$ wet mass of macrophytes and $2.5 \mathrm{~kg}$ dry mass of mixed leaf litter. Tanks were fertilized once before experiment initiation with nitrogen (10.4 $\mathrm{g} \mathrm{NaNO}_{3}$ per tank) and phosphorus (0.33 $\mathrm{g} \mathrm{NaH}_{2} \mathrm{PO}_{4}$ per tank) to increase initial primary productivity. To establish a complex pond community, we stocked tanks repeatedly before the start of the experiment with concentrated zooplankton and $1 \mathrm{~L}$ of a highly concentrated and diverse mix of small invertebrates (benthic and pelagic organisms) sifted from the vegetation and sediment of two local fishless ponds. In addition, we added a diverse range of larger invertebrates (including other predatory insects; see Supplement) and tadpoles from five anuran species collected from four local ponds (299 Bufo nebulifer, 75 Rana clamitans, 157 Hyla (versicolor and cinerea) per tank). Together with natural colonization of tanks (mostly beetles and chironomids), this created a highly diverse community with $>60$ morpho-species of vertebrates and invertebrates across all tanks (see Supplement). The experiment started on 3 June when the different $A$. junius stages were added to the tanks and ended after three weeks when the first $A$. junius larvae started emerging from the tanks.

\section{Ecosystem functions and properties}

To quantify treatment effects on the ecosystem functioning, we measured a range of ecosystem processes and different aspects of community structure (see Appendix A for full details). We calculated the decomposition constant ( $k$ values) from the exponential decay curve model estimated from the dry mass lost per day from leaf litter bags that were added at the beginning of the experiment. We also estimated net primary productivity (NPP) and respiration (R) calculated from diurnal oxygen cycles (Wetzel and Likens 2000, Downing and Leibold 2002). We estimated weekly changes in biomass for two dominant forms of primary producer in our ponds, periphyton (benthic algae) and phytoplankton (pelagic algae), from extracted chlorophyll $a$ following standard procedures (see Appendix A for full details).

We monitored tanks daily and collected all emerging invertebrates and vertebrates. At the end of the experiment, we sampled the pond communities using the following approach. We first used depth-integrated tube samplers to collect zooplankton samples. Then we took standardized subsamples of macro-invertebrate communities from the vegetation and benthos. Finally, we collected all macro-invertebrates $(>4 \mathrm{~mm})$ and amphibians remaining in the tanks. We then quantified animal biomass and community structure (benthic, vegetation, total) by counting, measuring, and weighing $>35500$ individuals from $>65$ species (see Supplement).

Community structure was analyzed for both the abundances and total biomass of species (see Appendix A for details). The goal of this analysis was to detect if A. junius stages have different effects on the community structure rather than the total abundance of species. Thus we used relative biomass or density of each species (i.e., proportion of total community biomass or density of an experimental pond) to test for differences in community structure across treatments. Finally, we analyzed changes in the size structure of the macroinvertebrate community by comparing square roottransformed abundance of individuals within $\log _{10}$ size classes based on dry mass. Analyses using different bin sizes gave similar results (not shown here), indicating that the analysis is robust to changes in bin size. 


\section{Statistical analysis}

Algae biomass samples were analyzed using repeatedmeasures ANOVA with the $A$. junius size class treatment as a fixed effect. The appropriate covariance structure for the repeated-measures analysis ("type $=\operatorname{arh}(1)$ " for phytoplankton and "type = un" for periphyton) was determined using the fitted model with the lowest AIC value (Littell et al. 1998). NPP and respiration were analyzed using one-way ANOVA with treatment as a fixed effect and change (final sample - first sample) in NPP or respiration as the dependent variable to account for variation in initial conditions among tanks. Decomposition rates $(k)$ were analyzed with one-way ANOVA with treatment as a fixed effect and average leaf litter mass loss per day as the dependent variable. We used a one-tailed $\log$-likelihood ratio $\chi^{2}$ test to test for random block effects (Littell et al. 2006). When block effects were not significant, block degrees of freedom were pooled with the error term degrees of freedom for the final analysis. Because of the large variation in primary producer abundance among tanks at the beginning of the experiment, some tanks were significant outliers (based on Studentized residual test with Bonferroni adjustments and interquartile range tests) and thus were removed from the analysis. To account for the missing replicates in analyses, the degrees of freedom were adjusted using the Satterthwaite procedure (Littell et al. 2006). All data met normality and heteroscedasticity assumptions and were carried out using the "proc mixed" procedure in SAS 9.3 (Littell et al. 2006).

We analyzed differences in the structure of communities among treatments using nonparametric, permutational multivariate statistics based on Bray-Curtis similarity metrics using the software PRIMER 6 (Clarke and Gorley 2006). First, we tested whether the variability in community structure (i.e., dispersion) differed among treatments using PERMDISP (Anderson 2006). Secondly, if treatments met the assumption of similar variances, we tested whether communities differed significantly among treatments using permutational multivariate analysis, PERMANOVA (Anderson 2001, McArdle and Anderson 2001). When block effects were not significant, block degrees of freedom were pooled with the error term degrees of freedom for the final analysis. Both permutation analyses were carried out using 999 permutations and based on centroids. Community structures were visualized with nonmetric multidimensional scaling plots (nMDs) using PRIMER 6. In general, there were no significant differences between the control and any of the Anax size treatments, largely because of the substantial variation in the control. Visual inspection of nMDS plots revealed that the control was typically at the center and spread evenly over the different size treatments. Thus, we focus our analyses here on our main question, i.e., whether Anax size classes differed in their effect on community structure; we provide the control only as a reference. We used structural equation modeling to look at relationships among ecosystem response variables. Finally, to test whether potential differences in average biomass among predator treatments were important in explaining the observed results, we first tested whether average Anax biomass differed among treatments and then tested whether the per-unit-biomass effect size differed among predator size treatments for all ecosystem processes (NPP, R, K) and animal and plant biomass (see Appendix B for details).

RESUlts

\section{Decomposition rates}

Decomposition rates ( $k$ values) did not differ among size treatments $\left(P=0.921, F_{3,20}=0.16\right)$. For large Anax (AL), $k=0.0138 \pm 0.0009 \mathrm{mg} / \mathrm{d}$ (mean $\pm \mathrm{SE}$ ); for medium Anax (AM), $k=0.0109 \pm 0.0003 \mathrm{mg} / \mathrm{d}$; for small Anax (AS), $k=0.0108 \pm 0.0009 \mathrm{mg} / \mathrm{d}$; for the control, $k=0.0107 \pm 0.0006 \mathrm{mg} / \mathrm{d})$.

\section{Ecosystem productivity and respiration}

Net primary productivity (NPP).- NPP significantly increased during the experiment in all treatments (all $P$ $<0.003$ ), but the relative increase differed significantly among treatments (Table 1, Fig. 1). NPP increased three times as much in ponds with large and medium-sized Anax compared to ponds with small Anax (post hoc comparison: AL vs. AS, $P=0.0082$; AM vs. AS, $P=$ 0.0147). The increase in NPP was, on average, slightly higher in the control compared to ponds with small Anax, but this was not significant $(P=0.4696)$

Respiration.-The change in respiration rates during the experiment differed significantly among treatments (Table 1) and showed a pattern similar to that for NPP (Fig. 1). Respiration rates increased seven and five times more in ponds with large and medium Anax, respectively, compared to ponds with small Anax (post hoc comparison: AL vs. AS, $P=0.002$; AM vs. AS, $P=$ 0.0094). Ponds with small Anax were also the only ponds that showed no significant increase in respiration rates over the experiment $\left(t_{1,19}=0.86, P=0.4020\right.$; Fig. 1).

\section{Primary producer biomass}

Phytoplankton.-Over the whole experiment (census 1 vs. census 3), phytoplankton biomass in ponds with small Anax decreased three times more than in ponds with large Anax $(t=2.10, P=0.0504)$ and 10 and 16 times more than in ponds with medium-sized Anax and the control respectively (AS vs. AM, $t=2.60, P=0.0150$; AS vs. Control, $t=2.72 P=0.0139$ ). However, how phytoplankton biomass changed over time also differed significantly among treatments (Table 1). Phytoplankton biomass did not change significantly over time in the control or in treatments that had received medium-sized predators (Anax) (planned contrasts; control, all $P>$ 0.6; AM, all $P>0.3$ ) (Fig. 2A). In contrast, phytoplankton biomass declined significantly between each census period in treatments with small Anax 
TABLE 1. Treatment effects on ecosystem processes, with size indicating the main treatment that manipulated presence of three size classes of the keystone predator, larvae of the dragonfly Anax junius.

\begin{tabular}{|c|c|c|c|c|}
\hline Process and effect & $F$ & df & $P$ & Treatment ranks \\
\hline \multicolumn{5}{|l|}{ Decomposition rate } \\
\hline Size & 0.16 & 3,20 & 0.921 & NS \\
\hline \multicolumn{5}{|l|}{ Change in respiration } \\
\hline Size & 5.75 & 3,19 & 0.0057 & $\mathrm{~L} \geq \mathrm{M}>\mathrm{C} \geq \mathrm{S}$ \\
\hline \multicolumn{5}{|l|}{ Change in NPP } \\
\hline Size & 4.24 & 3,19 & 0.0188 & $\mathrm{~L} \geq \mathrm{M}>\mathrm{C} \geq \mathrm{S}$ \\
\hline \multicolumn{5}{|c|}{ Change in phytoplankton } \\
\hline Size & 1.88 & $3,22.5$ & 0.1620 & $\mathrm{~S}>\mathrm{L}>\mathrm{M} \geq \mathrm{C}$ \\
\hline Time & 6.19 & $2,26.4$ & 0.0062 & \\
\hline Size $\times$ Time & 3.17 & $6,26.5$ & 0.0178 & $\mathrm{~S}>\mathrm{L}>\mathrm{M} \geq \mathrm{C}$ \\
\hline \multicolumn{5}{|l|}{ Change in periphyton } \\
\hline Size & 0.76 & $3,19.4$ & 0.5320 & $\mathrm{~S}>\mathrm{L}>\mathrm{C} \geq \mathrm{M}$ \\
\hline Time & 26.92 & $2,17.7$ & $<0.0001$ & \\
\hline Size $\times$ Time & 3.02 & $6,17.6$ & 0.0325 & $\mathrm{~S}>\mathrm{L}>\mathrm{C} \geq \mathrm{M}$ \\
\hline
\end{tabular}

Notes: Time indicates changes in the respective response variable across three sampling periods over the course of the experiment, and it may interact with size. Decomposition rate was estimated for the whole duration of the experiment. Phytoplankton and periphyton biomass were analyzed using repeated-measures ANOVA with the Satterthwaite procedure to adjust df to account for missing replicates. Change in respiration, NPP, periphyton, and phytoplankton indicates the change in the respective rate or biomass over the entire duration of the experiment. Treatment ranking indicates relative differences in average values among size classes of A. junius (small, $\mathrm{S}$; medium, M; large, L) and the control (C).

(planned contrasts; all $P<0.01$ ), whereas biomass only declined significantly between the second and third census period in treatments with large Anax (planned contrasts; first vs. second, $P=0.989$; second vs. third, $P$ $=0.011$ ).

Periphyton.- The change in periphyton biomass over time differed significantly among treatments (Table 1 , Fig. 2B). Periphyton biomass only declined significantly during the first and second census period in treatments with small Anax (planned contrasts; first vs. second, $P=$ 0.0002 ; second vs. third, $P=0.2897$ ), whereas it declined significantly between each census period in all other treatments including the control (planned contrasts; all $P<0.024)$. Furthermore, the magnitude in decline also varied among treatments during the first and second census period: phytoplankton declined twice as much in treatments with large and small Anax compared to treatments with medium Anax or the control (Fig. 2B). In general, these differences in temporal dynamics of phytoplankton and periphyton indicate that not only the quantitative but also the qualitative impact of Anax on both types of primary producer biomass differed significantly among size classes.

\section{Vertebrate and invertebrate biomass}

The final biomass of animals (vertebrates plus invertebrates) did not differ among ponds with small $(1991 \pm 315.5 \mathrm{mg}$, mean $\pm \mathrm{SE})$ and medium-sized (1942.6 $\pm 3.15 .5 \mathrm{mg})$ Anax, but both had $\sim 30 \%$ lower biomass than ponds with large Anax $(2689.2 \pm 223.1$ mg) (AL vs. AM, $P=0.03$; AL vs. AS, $P=0.04$; Fig. 3A). A separate analysis for habitat-specific biomass of macro-invertebrates (see Methods) revealed that Anax size classes also differed in their habitat-specific effects. Invertebrate biomass in the vegetation differed significantly $\left(F_{3,20}=6.38, P=0.003\right)$ among Anax size treatments (Fig. 3B). Average invertebrate biomass was 3.3 and 2.6 times lower in ponds with small Anax compared to ponds with large Anax or medium Anax, respectively. Interestingly, invertebrate biomass was two times higher in the large-Anax treatment compared to

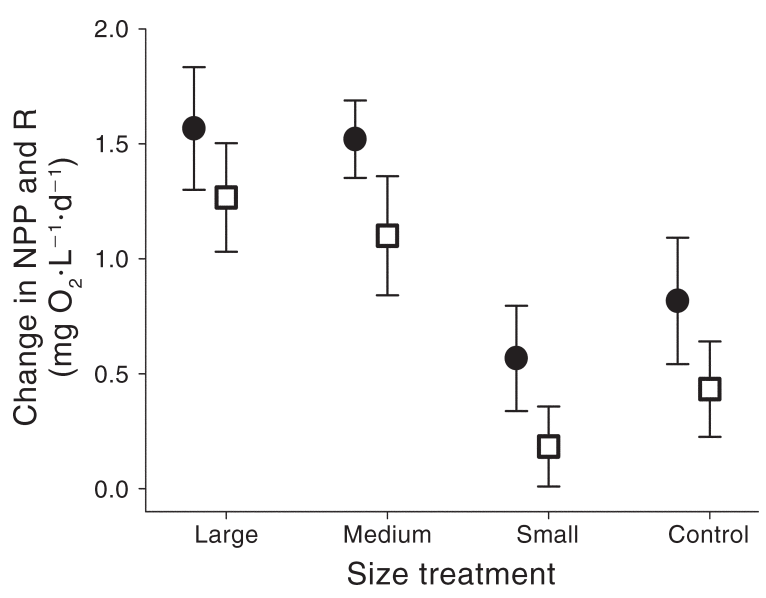

FIG. 1. Treatment effects on change (final minus initial; mean \pm SE) in ecosystem net primary productivity (NPP, solid circles) and respiration ( $\mathrm{R}$, open squares) during the experiment. Control indicates that no Anax junius dragonfly larvae were added; large, medium, and small indicate the respective size class of the predator $A$. junius that was added to experimental ponds. 

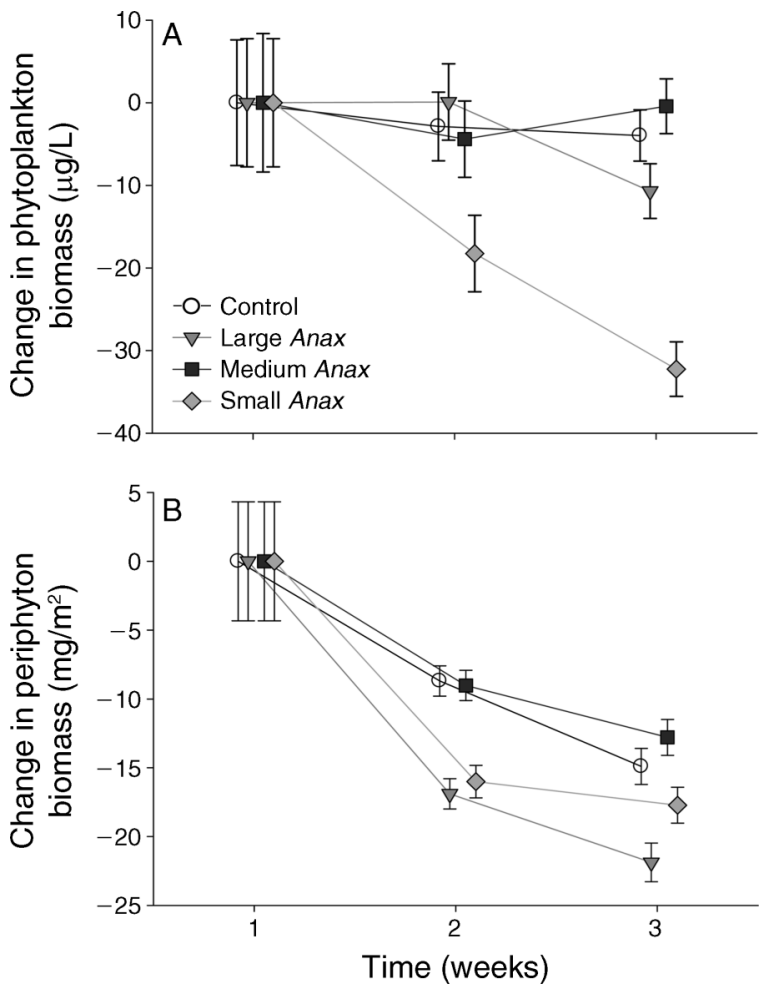

FIG. 2. Relative change in (A) phytoplankton biomass and (B) periphyton biomass measured from chlorophyll $a$ extractions across treatments over three weeks. Values are standardized by subtracting initial measurements at the beginning of the experiment from all subsequent observations of a given tank. Control indicates no added $A$. junius; large, medium, and small Anax indicate the respective size class of $A$. junius that was added to experimental ponds. Symbols indicate model estimated means $( \pm \mathrm{SE})$ that account for missing values and repeated measures of ponds.

the control $(P=0.0085)$, whereas small Anax treatments had half the invertebrate biomass observed in the control, although this difference was only significant when the significant outlier was removed from the small Anax treatment $(P=0.0166)$. Conversely, there were no differences among treatments in biomass for benthic invertebrates $\left(F_{3,15}=0.33, P=0.803\right)$. In general, this indicates that size classes of Anax differed in their microhabitat-specific effects on lower trophic levels.

\section{Community structure}

The overall community structure differed significantly among size treatments when analyzed based on abundance and biomass. Analyses based on abundance revealed that ponds with medium-sized Anax varied significantly more than ponds with small Anax (PERMDISP; $P=0.005$; Fig. 4A), whereas there were no differences among the other treatments (all $P>0.155$ ). The variance was lowest for small Anax, suggesting that they had the most consistent impact on community structure. In addition, the overall community structure differed significantly among ponds with small vs. large
Anax (PERMANOVA; pseudo- $F_{1,10}=2.606, P=0.008$; Fig. 4A). An analysis based on relative biomass showed no significant differences in variation in community structure among treatments (PERMDISP; all $P>$ 0.331). However, community structure of ponds also differed significantly among size class treatments (PERMANOVA; pseudo- $F_{2.15}=1.8518, P=0.041$ ), but in paired comparisons only the difference between medium and small Anax was significant (AM vs. AS, $P=0.011$; AM vs. AL, $P=0.570$; AL vs. AS, $P=0.210$; Fig. 4B). A separate analysis on only the zooplankton community structure showed similar patterns (not shown here). Because these analyses were based on proportional abundance (which corrects for differences in total densities or biomass), these results clearly indicate that different size classes of Anax had qualitatively different effects on the structure of the community, even after correcting for differences in effect size.

\section{Macro-invertebrate community size structure}

The size structure of the macro-invertebrate community differed significantly among Anax size class treatments (PERMANOVA; overall including block, pseudo- $F_{2,10}=2.79, P=0.038$; see Appendix C). The difference was largest among communities with large vs. small Anax (PERMANOVA; $P=0.048$ ), mostly because
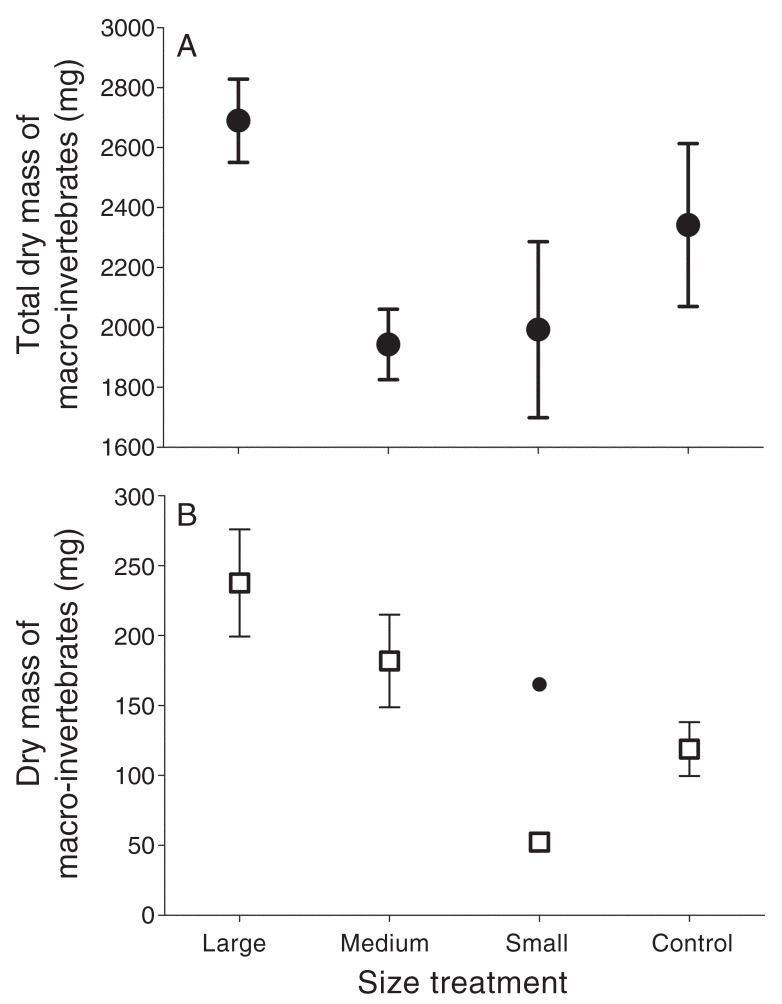

FIG. 3. (A) Total dry mass of macro-invertebrates and (B) biomass in the vegetation subsample in ponds without added $A$. junius (control) or with large, medium, or small stages of $A$. junius. All values are means \pm SE. The small solid circle in panel (B) indicates a significant outlier. 

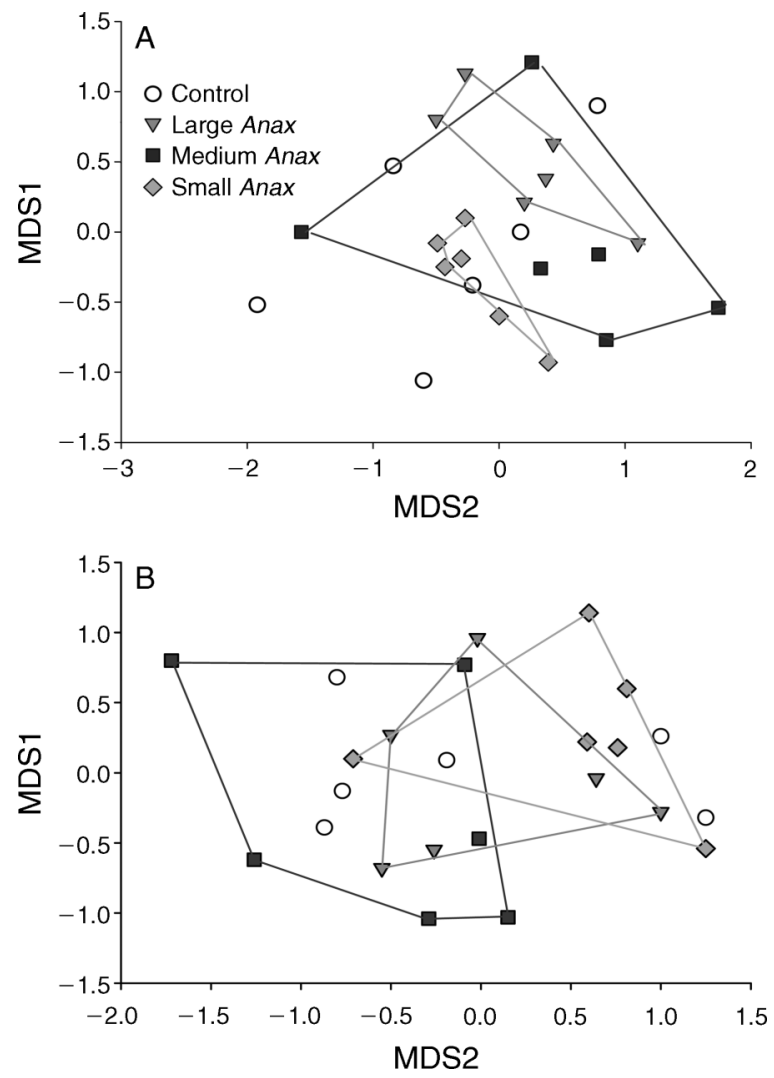

FIG. 4. Differences in community structure among ponds without added $A$. junius (control) or with large, medium, or small stages of $A$. junius. The figure presents nonmetric multidimensional scaling (nMDS) plots of species' relative (A) densities and (B) biomass (i.e., proportional abundance within a pond) in control and different Anax treatments. Points represent individual replicates of a treatment, and polygons contain all replicates within a treatment. The nMDS stress is: (A) 0.19, (B) 0.16 .

abundance within the smallest macro-invertebrate size class (0.001-0.01 mg) was fourfold lower in small-Anax compared to large-Anax ponds. The size structure of communities with medium-sized Anax was intermediate between communities with large or small Anax (AM vs. AL, $P=0.266$; AM vs. AS, $P=0.063$ ). There was no difference in the variance of community structure among treatments (PERMDISP; $P>0.328$ ).

\section{Relations among response variables}

To get more insight into the relationships among response variables, we carried out several analyses using structural equation modeling that connected relative changes in the community structure (based on two nMDS scores from Fig. 4) with changes in primary producer biomass and changes in NPP and respiration (see Appendix D for details). The analyses revealed that regardless of how community structure was analyzed (relative densities or relative biomass of species), one nMDS score was significantly negatively related to changes in periphyton biomass whereas the other was positively related to changes in phytoplankton biomass. A more detailed analysis suggests that the change in periphyton biomass was largely driven by changes in amphibian community structure (i.e., relative density of Hyla) among treatments. We suspected that differences in zooplankton community structure could be responsible for changes in phytoplankton biomass, but no simple relationships could be identified, suggesting that more complex interactions were responsible for this. Changes in NPP and respiration, in turn, were largely driven by changes in periphyton biomass, whereas changes in phytoplankton biomass or community structure had no significant effects. In general this indicates that the stage-specific effects of A. junius on primary producer biomass and ecosystem processes were not driven by direct effects, but rather by a range of complex indirect interactions (e.g., trophic cascades).

\section{Per-unit-biomass effect of predator stages}

It is possible that some of the observed quantitative differences in ecosystem properties (NPP, respiration, primary producer and animal biomass) among treatments could arise at least partially due to differences in predator biomass across treatments. To account for this potential confounding effect, we calculated average Anax biomass for a given predator stage treatment and used these estimates to calculate the per-unitbiomass effect of predator stages on these ecosystem processes (see Appendix B for details).

We found that the average Anax biomass across the experiment did not differ significantly (unequal variance $t$ test; $P=0.29)$ between small $(226.8 \pm 15.4 \mathrm{mg}$, mean \pm SE) and large predator stages $(264.3 \pm 22.2 \mathrm{mg})$, but both had 1.3-1.5 times lower Anax biomass (unequal variance $t$ test; AM vs. AL, $P=0.035$; AM vs. AS, $P<$ $0.001)$ than treatments with medium-sized Anax $(352.5$ $\pm 20.6 \mathrm{mg}$ ).

Overall, we found the same pattern for per-unitbiomass effects of predator stages as in our analyses of the untransformed data (see Appendix B for details). Per-unit-biomass effect on NPP, respiration, periphyton, phytoplankton and animal biomass, and macroinvertebrate biomass in the vegetation all differed significantly among size treatments, whereas decomposition and benthic macro-invertebrate biomass did not differ among treatments (Appendix B: Table B1, Figs. B1-B3). Again, the largest differences were observed between the per-unit-biomass effects of small and large predator stages, and the effect size often differed not only in magnitude but also direction (i.e., positive vs. negative effect relative to the control; see Appendix B: Table B1, Figs. B1-B3).

\section{DisCuSSION}

Ontogenetic niche shifts are ubiquitous in natural communities (Rudolf and Lafferty 2011), but little is known about whether and how they scale up to influence the structure and functioning of complex ecosystems. 
Our results clearly show that the impact of a predator on the structure of complex communities and ecosystem processes depends upon the ontogenetic stage of the predator. Importantly, these differences remained unchanged even after accounting for potential differences in predator biomass across treatments. In general this indicates clear functional differences among stages within a keystone species that can scale up to alter the functioning of entire ecosystems. Furthermore, the stage-specific effects did not simply scale with size or biomass of the predator, indicating ontogenetic niche shifts in species interactions. These results emphasize the importance of accounting for ontogenetic functional diversity within species for predicting how natural and anthropogenic changes alter natural ecosystems.

\section{Size, biomass, and functional differences among ontogenetic stages}

We found that altering the size structure of our focal species changed all aspects of the ecosystem, including ecosystem processes, primary producer and animal biomass, and community structure. This strong effect is even more impressive given the considerable natural variation in initial primary producer biomass and invertebrate colonization among experimental ponds. It is possible that simple biomass or size scaling relationships could create differences among predator stages in community- and ecosystem-level effects. Because we used natural densities for each stage, initial predator biomass was positively related with size, but biomass differences strongly attenuated over the experiment (because predator growth rates and mortality rates decreased with body size). As a consequence, average biomass of Anax during the experiment was not significantly different between large and small predator stages, but both had $\sim 1.3$ times lower biomass than treatments with medium-sized Anax (see Appendix B for details). However, small individuals have higher metabolic rates and thus higher per-biomass consumption rates. For example, in an aquatic shredder guild, perunit-biomass consumption was higher when it was composed of small stages compared to large stages (Reiss et al. 2011). Therefore, even though total predator biomass was similar in treatments with small and large predator stages, the potentially high per-biomass consumption rates of small predators could have resulted in effects comparable to or stronger than those of predators in the medium-sized and large Anax treatments.

Several patterns in our data suggest that observed differences cannot be explained by simple size-scaling relationships of consumption (metabolic) rates of the predator but instead indicate ontogenetic niche shifts in ecological interactions in a keystone predator. First, even after accounting for differences in predator biomass, we found that the per-unit-biomass effect differed among predator stages. Second, neither large nor small stages consistently had the strongest effect on all ecosystem response variables. For instance, although large stages had the strongest impact on NPP and respiration, small stages had up to 10 -fold stronger effects on primary producer biomass and reduced total animal biomass significantly more than large stages. Third, the ranking of size classes based on effect size did not consistently scale with size (positively or negatively) and varied depending on the response variable. For example, medium-sized stages had the weakest effect on primary producer biomass (despite having the highest predator biomass of all treatments) while small stages had the strongest effect. Finally, large and small stages often had opposing effects relative to the control, indicating clear qualitative differences in the nature of the effect. Similarly, the temporal dynamics of the biomass of both types of primary producers differed significantly among stages even after accounting for differences in total biomass of primary producers, again indicating clear qualitative differences. These qualitative differences, opposite effects, and changes in effect size ranking among stages are not predicted from simple differences in foraging or metabolic rates that scale with size, biomass, or a combination of the two factors.

Overall, our results indicate that stages differed significantly in their effect on community composition (for both biomass and densities). Because this analysis focused on proportional differences, significant treatment effects indicate that stages did not only alter the absolute abundance of species but also altered the relative abundance of species within a community. This clearly indicates that stages differed in their direct and indirect interactions within the food web, leading to a structural change in the community composition. Results from the path analysis suggest that these differences among stages in interactions indirectly led to concurrent changes in ecosystem processes such as NPP, respiration, or biomass of trophic levels.

The ontogenetic shift in species interactions in our system is consistent with diet shifts observed in other species of dragonfly larvae (e.g., Woodward and Hildrew 2002, Rudolf and Armstrong 2008), predatory invertebrates, and vertebrates (e.g., Polis 1984, Munoz and Ojeda 1998, Woodward and Hildrew 2002, Rudolf 2006). Such diet shifts are often correlated with ontogenetic shifts in microhabitat use (e.g., Persson and Eklov 1995, Rudolf and Armstrong 2008) and preference for different-sized prey (e.g., large predators often consume larger prey; Woodward et al. 2005), which could explain why small and large stages differed in their microhabitat-specific effects (i.e., invertebrate biomass in the vegetation) and effects on size structure of the macro-invertebrate community, respectively. However, our results extend these diet observations by demonstrating that differences among ontogenetic stages can lead to changes in the structure of complex communities and qualitatively different effects on other ecosystem properties. Thus, the functional role of a species is not constant, but instead changes with shifts in 


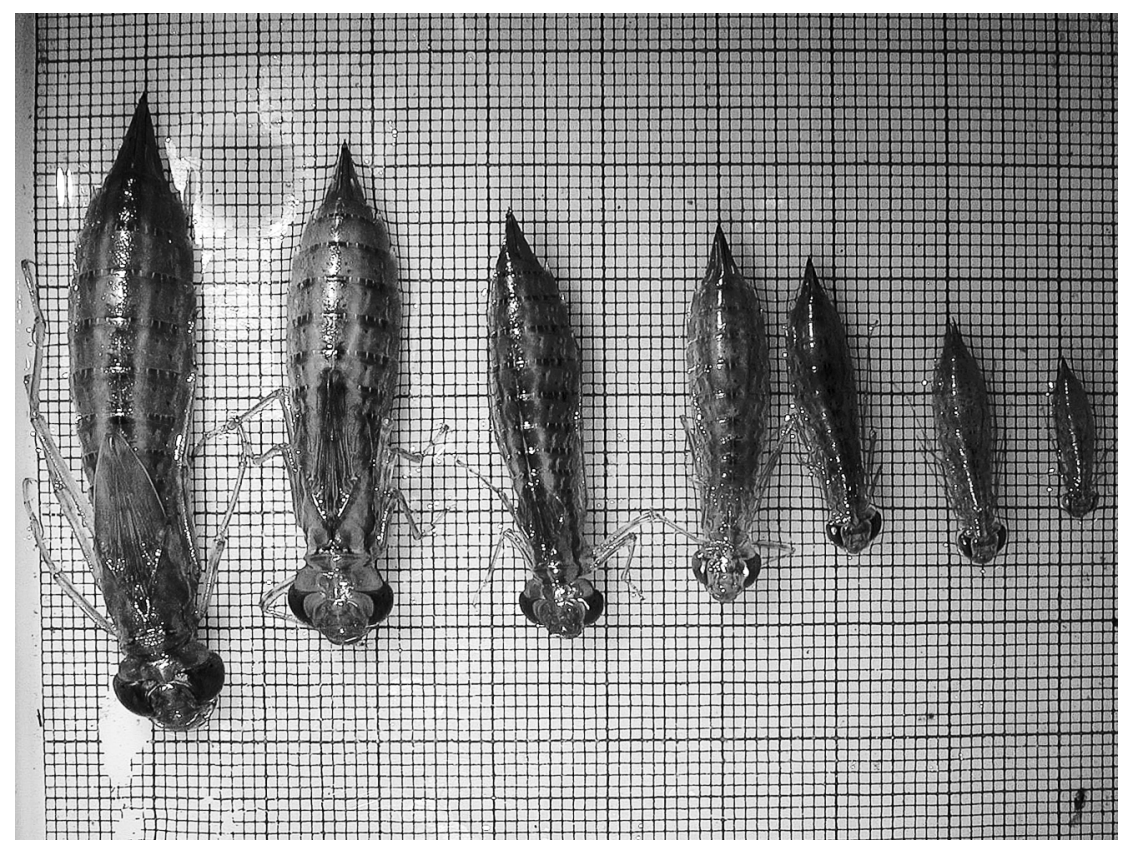

Plate 1. Example of size differences among successive developmental stages of the focal species Anax junius. The picture only shows the seven (out of 13) largest developmental stages, as the smallest self-feeding (youngest) stage have a body length of only a few millimeters. Photo credit: V. H. W. Rudolf.

the size structure of the species. Given that populations often consist of multiple stages, the next intriguing question is whether in such scenarios the functional role of a species is simply the sum of its individual components (stages), or whether more complex effects arise.

The results indicate that ontogenetic functional diversity is important at the community and ecosystem scale, but how can we predict the changes in community- and ecosystem-level processes that occur with changes in the ontogeny of organisms if they do not scale in a simple way with body size? Given the ontogenetic shifts in species interactions, it is not surprising that body size was a poor predictor for how individuals differed in their effect on the ecosystem. Body size arguably determines the strength of species interactions (e.g., an increase in predator-prey size ratio generally increases the effect of the predator on its prey), but differences in body size also lead to differences in presence/absence of species interactions (e.g., if the predator-prey size ratio increases enough, the predator might switch from one prey species to another). Thus, the potential for body size (mass) differences to predict differences in the net effect of individuals on complex community and ecosystem processes will decrease rapidly with increasing differences in interaction among individuals. Thus, we need a framework to predict how species interactions change over ontogeny. Recent studies suggest that optimal foraging theory can be used (to some extent) to predict the diet of consumers (and thus the structure of food webs) based on the relative body size differences of individuals within a community.
In combination with a size-based interaction strength, a similar optimal foraging approach potentially could be used within species to predict ontogenetic diet shifts, providing a more general framework to predict how individuals (among and within species) influence ecosystems. However, the question is whether this approach can be extended to explain intra- and interspecific functional differences, or whether we also need to account for the identity or traits of species (e.g., due to morphological constraints). Thus, future studies that examine whether functional differences among size classes are consistent across species and how ontogenetic functional diversity relates to species traits are needed to identify general patterns and develop a general predictive framework to ontogenetic functional diversity into community ecology.

\section{Ontogenetic functional diversity and natural ecosystems}

Although increasing evidence indicates that ontogenetic niche shifts can have important effects on the dynamics of species interactions (reviewed in Miller and Rudolf 2011), they have received surprising little attention at a community or ecosystem scale (but see Rudolf and Lafferty 2011). Our results indicate that ontogenetic niche shifts in a keystone predator can lead to functional differences among stages within populations. As a consequence, changes in the size structure of predator populations can result in concordant changes in ecosystem structure and processes. Given that ontogenetic niche shifts are ubiquitous in natural communities (Rudolf and Lafferty 2011), this has several important implications. First, our results indi- 
cate that the classical approach of assuming an average functional role of a species can be misleading, because its role is dynamic and will change with shifts in the size structure of the species. Secondly, it emphasizes the importance of accounting for bio- (functional) diversity below the species level to predict how natural and anthropogenic changes impact natural ecosystems.

While, to the best of our knowledge, our results are the first experimental demonstration of clear functional differences among ontogenetic stages at the ecosystem level, these findings are consistent with observational data from other systems. For example, in shallow lakes, dramatic shifts in the size structure of the top predator, perch, were correlated with shifts in the relative abundance of zooplankton and phytoplankton (Persson et al. 2003). Although this change in perch size structure was probably driven by size-structured interactions among stages within the population (cannibalism), many other natural or anthropogenic factors can also lead to shifts in the size structure of populations. For example, harvesting in fisheries is extremely size selective and has led to global decline in the average body size among top predatory fish (e.g., Shackell and Frank 2007, Swain et al. 2007). Recent studies indicate that although biomass remained largely unchanged, this decline was correlated with changes in abundances of lower trophic levels (Shackell et al. 2010). Both observations are consistent with our experimental findings that ontogenetic shifts in the functional role of species can alter the structure of communities. Our study provides an important next step by demonstrating that such shifts can also lead to changes in ecosystem processes. Given the high frequency of ontogenetic niche shifts in natural communities (Rudolf and Lafferty 2011), this emphasizes the importance of accounting for functional biodiversity below the species level (i.e., ontogenetic functional diversity) to predict how natural and anthropogenic changes alter the structure and functioning of natural ecosystems.

\section{ACKNOWLEDGMENTS}

We thank A. Dunham for discussion of the study, P. Delclos, L. Krenek, A. Roman, B. Van Allen, and B. Wise for help in the field and processing samples, and M. Gimmel for identifying beetles. All work was conducted according to IACUC protocol No. A09022601. The work was supported by NSF DEB-0841686 to V. H. W. Rudolf.

\section{Literature Cited}

Anderson, M. J. 2001. A new method for non-parametric multivariate analysis of variance. Austral Ecology 26:32-46.

Anderson, M. J. 2006. Distance-based tests for homogeneity of multivariate dispersions. Biometrics 62:245-253.

Benton, T. G., S. J. Plaistow, and T. N. Coulson. 2006. Complex population dynamics and complex causation: devils, details and demography. Proceedings of the Royal Society B 273:1173-1181.

Boege, K., and R. J. Marquis. 2005. Facing herbivory as you grow up: the ontogeny of resistance in plants. Trends in Ecology and Evolution 20:441-448.

Bolnick, D., R. Svanback, J. Fordyce, L. Yang, J. Davis, C. D. Hulsey, and M. Forister. 2003. The ecology of individuals: incidence and implications of individual specialization. American Naturalist 161:1-28.

Chalcraft, D. R., and W. J. Resetarits. 2004. Metabolic rate models and the substitutability of predator populations. Journal of Animal Ecology 73:323-332.

Clarke, K., and R. Gorley. 2006. PRIMER v6: User manual/ tutorial. PRIMER-E, Plymouth Marine Laboratory, Plymouth, UK. http://www.primer-e.com/index.htm

Downing, A. L., and M. A. Leibold. 2002. Ecosystem consequences of species richness and composition in pond food webs. Nature 416:837-841.

Duffy, J. E., B. J. Cardinale, K. E. France, P. B. McIntyre, E. Thebault, and M. Loreau. 2007. The functional role of biodiversity in ecosystems: incorporating trophic complexity. Ecology Letters 10:522-538.

Emmerson, M. C., and D. Raffaelli. 2004. Predator-prey body size, interaction strength and the stability of a real food web. Journal of Animal Ecology 73:399-409.

Harmon, L. J., B. Matthews, S. Des Roches, J. M. Chase, J. B. Shurin, and D. Schluter. 2009. Evolutionary diversification in stickleback affects ecosystem functioning. Nature 458:11671170.

Hooper, D. U., et al. 2005. Effects of biodiversity on ecosystem functioning: A consensus of current knowledge. Ecological Monographs 75:3-35.

Littell, R. C., P. R. Henry, and C. B. Ammerman. 1998. Statistical analysis of repeated measures data using SAS procedures. Journal of Animal Science 76:1216-1231.

Littell, R. C., G. A. Milliken, W. W. Stroup, R. D. Wolfinger, and O. Schabenberger. 2006. SAS for mixed models. Second edition. SAS Institute, Cary, North Carolina, USA.

Lomnicki, A. 1988. Population ecology of individuals. Princeton University Press, Princeton, New Jersey, USA.

Loreau, M., et al. 2001. Biodiversity and ecosystem functioning: current knowledge and future challenges. Science 294:804808 .

McArdle, B. H., and M. J. Anderson. 2001. Fitting multivariate models to community data: a comment on distance-based redundancy analysis. Ecology 82:290-297.

McCann, K. S. 2000. The diversity-stability debate. Nature 405:228-233.

McPeek, M. A. 1998. The consequences of changing the top predator in a food web: a comparative experimental approach. Ecological Monographs 68:1-23.

Miller, T. E. X., and V. H. W. Rudolf. 2011. Thinking inside the box: community-level consequences of stage-structured populations. Trends in Ecology and Evolution 26:457-466.

Mittelbach, G. G., and L. Persson. 1998. The ontogeny of piscivory and its ecological consequences. Canadian Journal of Fisheries and Aquatic Sciences 55:1454-1465.

Munoz, A. A., and F. P. Ojeda. 1998. Guild structure of carnivorous intertidal fishes of the Chilean coast: implications of ontogenetic dietary shifts. Oecologia 114:563-573.

O'Connor, N. E., J. H. Grabowski, L. M. Ladwig, and J. F. Bruno. 2008. Simulated predator extinctions: Predator identity affects survival and recruitment of oysters. Ecology 89:428-438.

Osenberg, C. W., G. G. Mittelbach, and P. C. Wainwright. 1992. Two-stage life histories in fish: The interaction between juvenile competition and adult performance. Ecology 73:255267.

Persson, L. 1999. Trophic cascades: abiding heterogeneity and the trophic level concept at the end of the road. Oikos 85:385-397.

Persson, L., A. M. De Roos, D. Claessen, P. Bystrom, J. Lovgren, S. Sjogren, R. Svanback, E. Wahlstrom, and E. Westman. 2003. Gigantic cannibals driving a whole-lake trophic cascade. Proceedings of the National Academy of Sciences USA 100:4035-4039. 
Persson, L., and P. Eklov. 1995. Prey refuges affecting interactions between piscivorous perch and juvenile perch and roach. Ecology 76:70-81.

Polis, G. A. 1984. Age structure component of niche width and intraspecific resource partitioning: Can age groups function as ecological species? American Naturalist 123:541-564.

Power, M. E., D. Tilman, J. A. Estes, B. A. Menge, W. J. Bond, L. S. Mills, G. Daily, J. C. Castilla, J. Lubchenco, and R. T. Paine. 1996. Challenges in the quest for keystones. BioScience 46:609-620.

Reiss, J., R. A. Bailey, D. M. Perkins, A. Pluchinotta, and G. Woodward. 2011. Testing effects of consumer richness, evenness and body size on ecosystem functioning. Journal of Animal Ecology 80:1145-1154.

Reiss, J., J. R. Bridle, J. M. Montoya, and G. Woodward. 2009. Emerging horizons in biodiversity and ecosystem functioning research. Trends in Ecology and Evolution 24:505-514.

Rudolf, V. H. W. 2006. The influence of size-specific indirect interactions in predator-prey systems. Ecology 87:362-371.

Rudolf, V. H. W. 2012. Seasonal shifts in body size diversity and trophic interactions in size-structured predator-prey systems. Journal of Animal Ecology 81:524-523.

Rudolf, V. H. W., and J. Armstrong. 2008. Emergent impacts of cannibalism and size refuges in the prey on intraguild predation systems. Oecologia 157:675-686.

Rudolf, V. H. W., and K. D. Lafferty. 2011. Stage structure alters how complexity affects stability of ecological networks. Ecology Letters 14:75-79.

Schneider, F. D., S. Scheu, and U. Brose. 2012. Body mass constraints on feeding rates determine the consequences of predator loss. Ecology Letters 15:436-443.

Shackell, N. L., and K. T. Frank. 2007. Compensation in exploited marine fish communities on the Scotian Shelf, Canada. Marine Ecology Progress Series 336:235-247.

Shackell, N. L., K. T. Frank, J. A. D. Fisher, B. Petrie, and W. C. Leggett. 2010. Decline in top predator body size and changing climate alter trophic structure in an oceanic ecosystem. Proceedings of the Royal Society B 277:13531360.

Swain, D. P., A. F. Sinclair, and J. Mark Hanson. 2007. Evolutionary response to size-selective mortality in an exploited fish population. Proceedings of the Royal Society B 274:1015-1022.

Urban, M. 2007. Predator size and phenology shape prey survival in temporary ponds. Oecologia 154:571-580.

Van Buskirk, J. 1988. Interactive effects of dragonfly predation in experimental pond communities. Ecology 69:857-867.

Werner, E. E. 1988. Size, scaling and the evolution of complex life cycles. Pages 60-81 in B. Ebenman and L. Persson, editors. Size-structured populations. Springer, New York, New York, USA.

Werner, E. E., and J. F. Gilliam. 1984. The ontogenetic niche and species interactions in size structured populations. Annual Review of Ecology and Systematics 15:393-425.

Wetzel, R. G., and G. E. Likens. 2000. Limnological analyses. Third edition. Springer, New York, New York, USA.

Wilbur, H. M. 1980. Complex life-cycles. Annual Review of Ecology and Systematics 11:67-93.

Wilbur, H. M., and J. E. Fauth. 1990. Experimental aquatic food webs: interactions between two predators and two prey. American Naturalist 135:176-204.

Wissinger, S. A. 1988. Life history and size structure of larval dragonfly populations. Journal of North American Benthological Society 7:13-28.

Wissinger, S. A. 1992. Niche overlap and the potential for competition and intraguild predation between size-structured populations. Ecology 73:1431-1444.

Woodward, G., B. Ebenman, M. Emmerson, J. M. Montoya, J. M. Olesen, A. Valido, and P. H. Warren. 2005. Body size in ecological networks. Trends in Ecology and Evolution 20:402-409.

Woodward, G., and A. G. Hildrew. 2002. Body-size determinants of niche overlap and intraguild predation within a complex food web. Journal of Animal Ecology 71:10631074.

Yang, L. H., and V. H. W. Rudolf. 2010. Phenology, ontogeny, and the effects of climate change on the timing of species interactions. Ecology Letters 13:1-10.

Yodzis, P., and S. Innes. 1992. Body size and consumerresource dynamics. American Naturalist 139:1151-1175.

\section{Supplemental Material}

Appendix A

Detailed description of the experimental methods (Ecological Archives E094-093-A1).

Appendix B

Detailed description of biomass-corrected analyses and results (Ecological Archives E094-093-A2).

Appendix C

Average abundance-biomass relationships in different predator treatments (Ecological Archives E094-093-A3).

\section{Appendix D}

Results of structural equation modeling indicating relationships among treatments and ecosystem variables (Ecological Archives E094-093-A4).

\section{Supplement}

Treatment means and standard errors for densities and biomass of community members at the end of the experiment, categorized by taxa (Ecological Archives E094-093-S1). 